\title{
Screening of hepatocyte proteins binding with the middle surface protein of the hepatitis B virus by the yeast two-hybrid system
}

\author{
ZHIQUN LI ${ }^{1}$, ENQIANG LINGHU ${ }^{1}$ and $\mathrm{JUN} \mathrm{CHENG}^{2}$ \\ ${ }^{1}$ Department of Gastroenterology and Hepatology, Chinese PLA General Hospital, Beijing 100853; \\ ${ }^{2}$ Institute of Infectious Diseases, Ditan Hospital, Capital Medical University, Beijing 100015, P.R. China
}

Received September 21, 2013; Accepted February 20, 2014

DOI: $10.3892 / \mathrm{mmr} .2014 .2069$

\begin{abstract}
The effect of the middle hepatitis B virus surface protein (MHBs) remains to be elucidated. To investigate the biological function of the MHBs protein, the present study performed yeast two-hybrid screening to search for proteins that interact with the MHBs protein in hepatocytes. The bait plasmid expressing the MHBs protein was constructed by cloning the gene of the MHBs protein into pGBKT7, then the recombinant plasmid DNA was transformed into AH109 yeast (a type). The transformed yeast AH109 was mated with yeast Y187 ( $\alpha$ type) containing the liver cDNA library plasmid in $2 \mathrm{X}$ yeast peptone dextrose adenine (YPDA) medium. The mated diploid yeast was plated on quadruple dropout medium (SD/-Trp-Leu-His-Ade) containing $X$ - $\alpha$-gal for selection and screening. Following extracting and sequencing of the plasmids from positive (blue) colonies, the sequence analysis was conducted and analyzed by bioinformatics methods. Two colonies were selected and sequenced. Among them, one was the human DNA sequence from the clone RP11-490D19 on chromosome 9 and the other was homo sapiens $12 \mathrm{BAC}$ RP11-180M15 (Roswell Park Cancer Institute Human BAC Library). The yeast two-hybrid system is an effective method for identifying hepatocyte proteins that interact with MHBs. The MHBs protein binds with different proteins suggesting that it has multiple functions in vivo.
\end{abstract}

Correspondence to: Dr Jun Cheng, Institute of Infectious Diseases, Ditan Hospital, Capital Medial University, 8 Jing Shun Street, Chaoyang, Beijing 100015, P.R. China

E-mail: jun.cheng.ditan@gmail.com; fairydoctor168167@163.com

Abbreviations: HBV, hepatitis B virus; MHBs, middle hepatitis B virus surface protein; LHBs, large hepatitis B virus surface protein; $\mathrm{MHBs}^{\mathrm{t}}$, C-terminally truncated middle size surface proteins; ORF, open reading frame; ER, endoplasmic reticulum; PCR, polymerase chain reaction; DNA-BD, DNA binding domain; DNA-AD, DNA activation domain; MBP1, MHB-binding protein 1; RT-PCR, reverse transcription-polymerase chain reaction; HCC, hepatocellular carcinoma; QDO, quadruple dropout medium lacking leucine, tryptophan, histidine and adenine

Key words: hepatitis B virus, MHBs, yeast two-hybrid

\section{Introduction}

Hepatitis B virus (HBV) DNA does not integrate at specific sites of the host genome. Thus, a common cis effect onto flanking cellular genes can be excluded as a general mechanism of HBV-associated carcinogenesis. However, integrated HBV DNA can encode two types of transcriptional activators: The already studied HBx and the PreS2 activators, including the large HBV surface protein (LHBs) and the C-terminally truncated middle size surface proteins $\left(\mathrm{MHBs}^{\mathrm{t}}\right)$ (1-3). The sequence encoding the PreS2 activators is localized on the HBV surface gene. The surface gene consists of a single open reading frame (ORF) divided into three coding regions: preS1, preS2 and S, each starting with an in-frame ATG codon. Through alternate translation initiation at each of the three AUG codons, a large (LHB; PreS1 + PreS2 + S), a middle size (MHB; PreS2 + S) and a small (SHB; S) envelope glycoprotein is able to be synthesized. The activator function of the surface protein requires the cytoplasmic orientation of the PreS2 domain (the minimal functional unit) that occurs in the case of $M_{H B}{ }^{t}$ and in a fraction of LHBs $(4,5)$. By contrast, full-length MHBs exhibit no transcriptional activator function: The PreS2 domain is directed into the lumen of the endoplasmic reticulum (ER). To investigate the biological importance of MHBs, the present study screened and identified the proteins interacting with MHBs with the yeast two-hybrid system 3 to elucidate the biological function of MHBs.

\section{Materials and methods}

Agents and culture media, yeast strains and plasmids. Taq DNA polymerase, T4 DNA ligase, and EcoRI and BamHI restriction endonucleases were purchased from Takara Bio, Inc. (Dalian, China). Lithium acetate, semi-sulfate adenine, acrylamide and N,N'-bis-acrylamide were purchased from Sigma (St. Louis, MO, USA). TEMED was obtained from Boehringer Mannheim GMBH (Mannheim, Germany). Tryptone and yeast extracts were purchased from Oxoid (Lenexa, KS, USA). X- $\alpha-G a l$ and Yeast peptone dextrose adenine (YPDA), $\mathrm{SD} /$-Trp SD/-Leu, SD/-Trp/-Leu, SD/-Trp/-Leu/-His and $\mathrm{SD} /$-Trp/-Leu/-His/-Ade culture media were obtained from Clontech Laboratories, Inc. (Mountain View, CA, USA). Protein-G agarose was obtained from Roche Diagnostics (Indianapolis, IN, USA). The pGEM-T vector was purchased 
from Promega Corporation (Madison, WI, USA). Yeast strains and plasmids for yeast two-hybrid experiments were obtained from Clontech Laboratories, Inc. as components of the Matchmaker two hybrid system 3. The yeast strain AH109

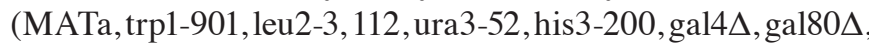
LYS2: GAL1 $1_{\mathrm{UAS}}-\mathrm{GAL1}_{\mathrm{TATA}^{-}}-\mathrm{HIS} 3, \mathrm{GAL}_{\mathrm{UAS}}-\mathrm{GAL} 2_{\mathrm{TATA}}-\mathrm{ADE} 2$, URA3: MEL1 $1_{\mathrm{UAS}}-\mathrm{MEL1}_{\mathrm{TATA}}$-LacZ) containing pGBKT7-53, coding for the DNA binding domain (DNA-BD)/mouse p53 fusion protein and AH109 used for cloning of bait plasmid, yeast strain Y187 (MATa ura3-52, his3-200,

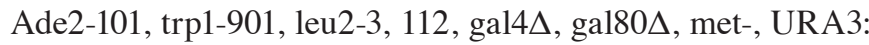
$\mathrm{GAL1}_{\mathrm{UAS}}-\mathrm{GAL1}_{\mathrm{TATA}}-$ lacZ MEL1) containing pTD1-1, in which pACT2 codes for the activation domain (AD)/SV40 large T antigen fusion protein and Y187 were used for the cloning of library plasmids. Pretransformed human cDNA liver cell library yeast strain (Y187). The bacterial strain DH5 $\alpha$ was used for cloning every shuttle plasmid. The yeast Escherichia coli shuttle plasmids pGBKT7 DNA-BD cloning plasmid, pGADT7 AD cloning plasmid, pGBKT7-53 control plasmid, pGADT7, pGBKT7-Lam control plasmid and the pCL1 plasmid were purchased from Clontech Laboratories, Inc. (K1612-1). The pGEM T vector was obtained from Promega Corporation.

The A7 plasmid containing the whole sequences of the adr subtype of HBV, was conserved by the Gene Laboratory of the Institute of Infectious Diseases in Ditan Hospital (Beijing, China).

Construction of the bait plasmid and the expression of the MHBs protein. The MHBs sequences were generated by polymerase chain reaction (PCR) amplification of the A7 plasmid (HBV strain adr). The A7 plasmid contains coding sequences for all of the HBV. The sequences of the primers, which contain the EcoRI and BamHI restriction enzyme sites, were as follows: Forward: 5'-GAATTCATGGTCACCTTGAGGTGG-3' and reverse: 5'-GGATCCAGTTTACATATGGGTTTCTG-3'. The PCR conditions were as follows: $94^{\circ} \mathrm{C}$ for $4 \mathrm{~min}, 94^{\circ} \mathrm{C}$ hot denaturalization for $50 \mathrm{sec}, 58^{\circ} \mathrm{C}$ annealing for $50 \mathrm{sec}$ and $72^{\circ} \mathrm{C}$ extension for $1 \mathrm{~min}$ for 35 cycles. The PCR product $(6 \mu \mathrm{l})$ was cloned with the pGEM-T vector. The primary structure of the insert was confirmed by direct sequencing. The fragment encoding MHBs was released from the pGEM-T-MHBs by digestion with EcoRI and BamHI, and ligated to pGBKT7. Vector pGBKT7 expressing proteins were fused with amino acids 1-147 of the GAL4 DNA-BD and pGADT7 expressing proteins were fused with amino acids 768-881 of the GAL4 AD. The plasmid pGBKT7-MHBs (Fig. 1) containing the full-length MHBs gene was able to direct the expression of the DNA-BD, c-myc and MHBs fusion protein. The plasmid was transformed into the AH109 yeast strain using the lithium acetate method (6). Transformed AH109 (bait) was cultured on QDO to exclude the auto-activation activity.

Yeast two-hybrid screening of the liver cell cDNA library. One large (2-3 mm), fresh (<2 months old) colony of AH109 (bait) was inoculated into $50 \mathrm{ml} \mathrm{SD} /$-Trp and incubated at $30^{\circ} \mathrm{C}$ overnight (16-24 h) with agitation at 250-270 rpm. Then, the cells were spun by centrifuging the entire $50 \mathrm{ml}$ culture at $1,000 \mathrm{x} \mathrm{g}$ for $5 \mathrm{~min}$. Following decanting the supernatant, the cell pellet was resuspended in the residual liquid by vortexing. A human liver cDNA library cloned into pACT2 and yeast reporter

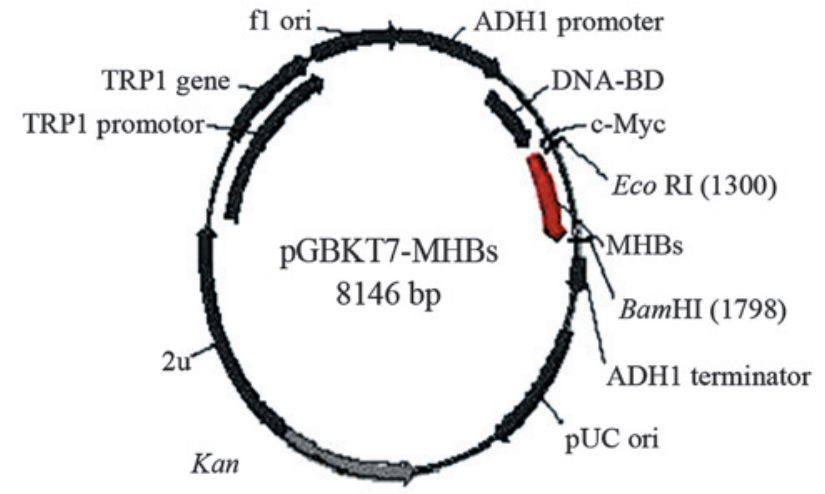

Figure 1. Structure of the bait plasmid pGBKT7-MHBs. DNA-BD, DNA binding domain; MHBs, middle surface protein of hepatitis B virus.

strain Y187 were co-cultured. The entire AH109 (bait) culture and the $1 \mathrm{ml}$ human liver cDNA library $\left(1 \times 10^{6} \mathrm{cfu} / \mathrm{ml}\right)$ were combined and cultured in a 2-1 sterile flask and $45 \mathrm{ml}$ of $2 \mathrm{X}$ YPDA/Kan was added and mixed gently. Following $20 \mathrm{~h}$ of mating, the cells were spun down and resuspended, and then were spread onto 50 large $(150 \mathrm{~mm})$ plates, containing $100 \mathrm{ml}$ of SD/-Ade/-His/-Leu/-Trp (QDO). Following 6-18 days of growth, the yeast colonies were transferred onto the plates containing X- $\alpha$-gal and blue colonies identified the expression of the MEL1 reporter gene. Approximately $1 \times 10^{6}$ colonies were screened and positive clones were identified. The yeast plasmid was isolated from positive yeast colonies using the Lyticase method (provided by Clontech Laboratories, Inc.), and transformed into super competent $E$. coli DH5 $\alpha$ using a chemical method. Transformants were plated on ampicillin super optimal broth selection media and grown under selection. Subsequently, pACT2-cDNA constructs were re-isolated, by $B g l$ II restriction enzyme digests and following sequencing of the positive colonies, the sequences were BLASTed with GenBank (http://blast.ncbi.nlm.nih.gov/Blast.cgi) to analyze the function of the genes.

To verify the true protein-protein interaction, the plasmids of positive colonies were transformed into the Y187 yeast strain, and false positives were excluded. Next, mating experiments were performed by mating with the AH109 yeast strain containing pGBKT7-MHBsor pGBKT7-Lam. Following mating, the diploid yeast were plated on SD/-Ade-His-Leu-Trp (QDO) covered with $\mathrm{X}$ - $\alpha$-gal to assess the specificity of interactions. Since plasmid pACT2-cDNA contains two restriction endonuclease sites of $\mathrm{BglII}$ on the two sides of multiple cloning sites, the gene fragments of different lengths verified that these screened clones were positive colonies.

\section{Results}

Identification of the plasmid and analysis of the cDNA sequence and homology. The MHBs gene was amplified by PCR from the plasmid A7 containing the whole fragment of the adr subtype of HBV and the PCR product was cloned into the pGEM-T vector. Analysis of the PCR products by agarose gel electrophoresis demonstrated that the clear bands were the expected size 846 bp of MHB. Following cutting MHBs-pGEM-T by EcoRI and BamHI restriction enzymes, 

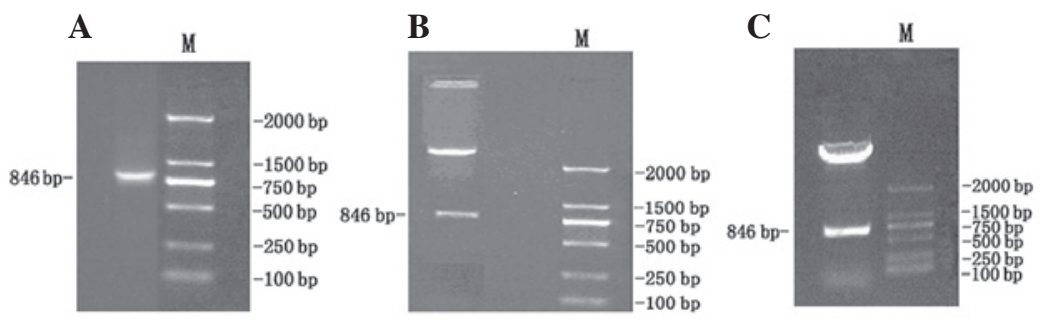

Figure 2. Construction and identification of the pGEM-T-MHBs plasmid by agarose gel electrophoresis. (A) MHBs gene amplified by PCR; (B) Restriction enzyme digestion of pGEM-T-MHBs plasmid with EcoRI/BamHI; (C) Restriction enzyme digestion of pGBKT7-MHBs plasmid with EcoRI/BamHI. M, DL2000 DNA Marker; MHBs, middle surface protein of hepatitis B virus.
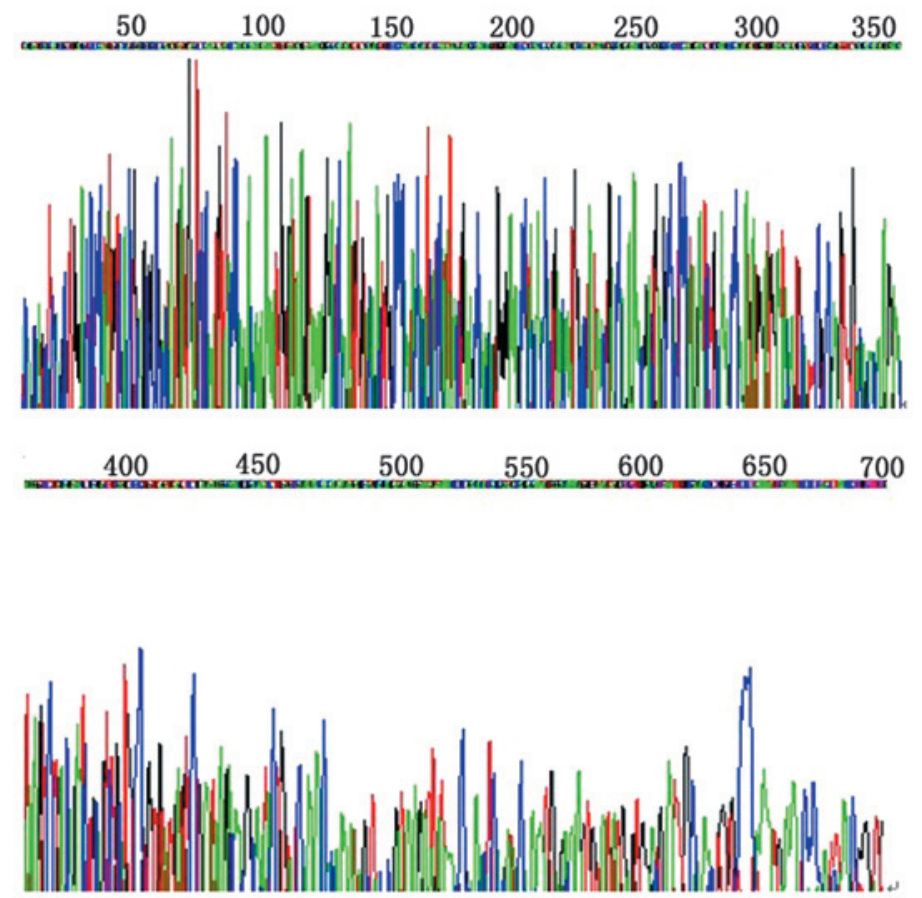

Figure 3. Sequence of the pGEM-T-MHBs plasmid by direct sequencing. MHBs, middle surface protein of hepatitis B virus.

MHBs were ligated in-frame into the yeast expression plasmid pGBKT7 EcoRI/BamHI sites using the yeast two-hybrid system 3. Restriction enzyme digestion of the pGBKT7-MHBs plasmid with EcoRI/BamHI yielded two bands: 7,300 bp empty pGBKT7 and 846 bp MHBs(Fig. 2A, B and C). The primary structure of the insert was confirmed by direct sequencing of the pGEM-T-MHBs sequence (Fig. 3).

Screening of the liver cell cDNA library and identification of the plasmid. The plasmids from the blue colonies containing only pGBKT7-MHBs were isolated as the bait for screening the human liver cell cDNA library. Positive clones interact with the MHBs protein growing on media containing $\mathrm{X}-\alpha-\mathrm{Gal}$ and lacking leucine, tryptophan, histidine and adenine (QDO). A total of two positive colonies were grown on the $\mathrm{X}-\alpha$-gal/QDO medium and expressed (blue colonies). The two colonies were prescreened by $B g l$ II digestion to ensure that only colonies with different inserts were subjected to sequencing. One colony was a novel gene with unknown function and interacted with MHBs in hepatocytes. The protein was designated MHB-binding protein 1 (MBP1). The MBP1 gene was amplified by the reverse transcription-polymerase chain reaction (RT-PCR) technique using HepG2 cDNA as a template and was inserted into the pGEM-T vector by TA cloning. In addition, analysis of the RT-PCR products by agarose gel electrophoresis demonstrated the clear bands with the expected size 201 bp of MBP1 (Fig. 4).

Analysis of cDNA sequencing and homology. Two colonies from the hepatocyte cDNA library were sequenced. Sequence homology searches in GenBank revealed that it was a novel gene and was designated MBP1. This cDNA was 201 bases long, which contained an ORF able to encode a protein of 66 amino acids (Fig. 5). The full-length sequences accepted by GenBank were obtained using the BLAST program at the National Center for Biotechnology Information. The GenBank accession number is DQ307498. A summary of the data is presented in Table I.

\section{Discussion}

HBV infection is the main factor that induces hepatocellular carcinoma (HCC) (7). An alternative underlying pathogenic mechanism of chronic infections and hepatocarcinogenesis 
Table I. Comparison between positive clones and similar sequences in GenBank.

High similarity to known genes

Number of similar $(\%)$

Homology $(\%)$

Human DNA sequence from clone RP11-490D19 on chromosome 9

1

99

Homo sapiens 12 BAC RP11-180M15 (Roswell Park

100

Cancer Institute Human BAC Library) complete sequence

BAC, bacterial artificial chromosomes.

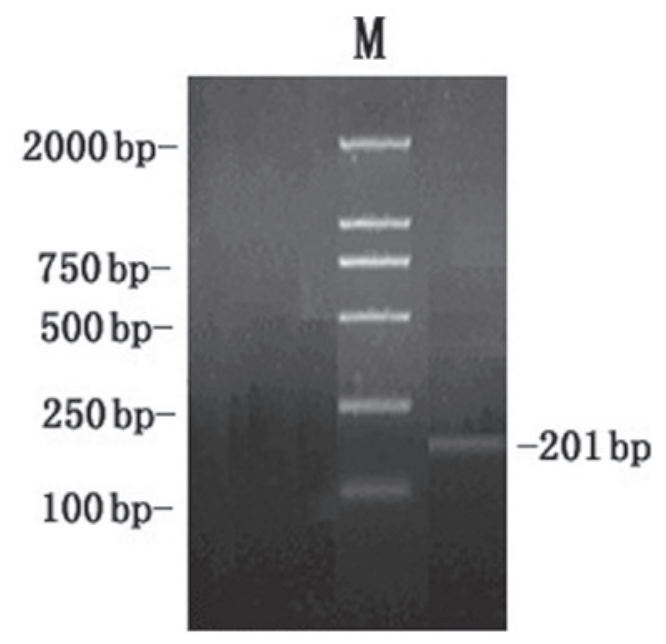

Figure 4. Analysis of the reverse transcription-polymerase chain reaction product MBP1 by agarose gel electrophoresis. M, DL2000 DNA Marker; 1, MBP1. MBP1, MHBs-binding protein 1.

may be the key step to mutual interaction between viral proteins and hepatocellular proteins, and this action may mediate viral entry into liver cells and affect the activities and function of these proteins. Furthermore, the protein from hepatocytes infected with HBV inversely disturbs viral replication and reduces immunity of the host, resulting in chronic liver diseases and HCC (8). The length of the nucleotide sequence of $\mathrm{HBV}$ is 3,182 nt and the main serum type is adr in China (9). The role of MHBs in the etiology of HCC is not well understood. Screening of hepatocyte proteins binding with MHBs by the yeast two-hybrid system 3 may aid in elucidating its role. The present study obtained a total of two positive colonies grown on the selective $\mathrm{SD} /$-Trp-Leu-His-Ade/X- $\alpha$-gal medium, one was the human DNA sequence from the clone RP11-490D19 on chromosome 9. In total, 15 loci on chromosome 9p and 17 were analyzed to clarify the involvement of a loss of heterozygosity (LOH) in HCC in Chinese patients positive for HBV and/or hepatitis $\mathrm{C}$ virus infection. The expression of tumor suppressor genes, including p53, p16 and the p15 gene was revealed to be correlated with a deletion of these genes. A high frequency of LOH was detected on chromosome 9p24 at locus D9S54 $(61.8 \%)$ and 9p21. No significant association between $\mathrm{LOH}$ and HCC clinico-pathological outcomes was observed. A high frequency of LOH occurs on chromosomes 9p and 17 in HCC in Chinese patients. Such sites may contain several putative tumor suppressor genes critically involved in the development and/or progression of HCC (10).

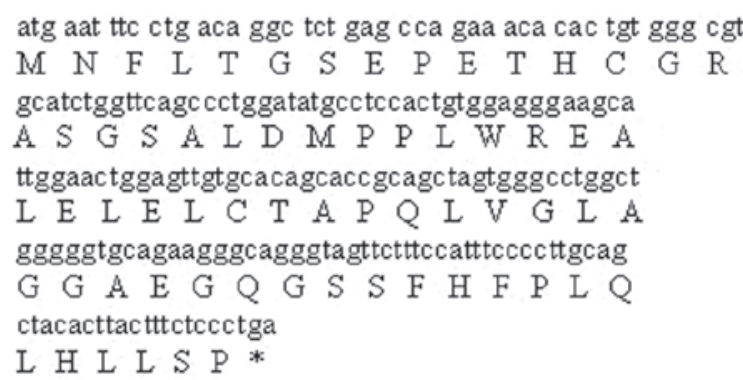

Figure 5. Nucleic and amino acid sequences of MHBs-binding protein 1 (GenBank: DQ307498).

The other positive colony was homo sapiens 12 BAC RP11-180M15 (Roswell Park Cancer Institute Human BAC Library, Buffalo, NY, USA). The end sequences from bacterial artificial chromosomes (BACs) provide highly specific sequence markers in large-scale sequencing projects. To date, $>300,000$ end sequences have been generated from $>186,000$ human BAC clones with an average read length of $>460 \mathrm{bp}$ for a total of $141 \mathrm{Mb}$ covering $\sim 4.7 \%$ of the genome. Over $60 \%$ of the clones have BAC end sequences (BESs) from the two ends representing $>5$-fold coverage of the human genome by the paired-end clones. The quality assessments and sequence analyses indicate that BESs from human BAC libraries developed at The California Institute of Technology (CalTech; Pasadena, CA, USA) and Roswell Park Cancer Institute exhibit similar properties. The analyses have highlighted differences in the insert size for different segments of the CalTech library (11).

The characterization of the interaction pattern of a protein could provide considerable assistance in the elucidation of the functions of that protein (12). The interactions between viral proteins and hepatocellular proteins are important in the pathogenesis of the virus and may mediate virus entry into hepatocytes. Yeast two-hybrid 3 is an effective gene analysis method, which is able to analyze the interactions between protein and protein, protein and DNA, and protein and RNA in eukaryotic cells. In addition, it is a novel genetics technique for investigating the interactions of proteins in physiological conditions in vivo.

The present study used the yeast two-hybrid system 3 based on the system originally designed by Fields and Song (13) and took advantage of the properties of the GAL4 protein of the yeast Saccharomyces cerevisiae. The GAL4-yeast two-hybrid assay uses two expression vectors, one uses GAL4-DNA-BD and the other uses GAL4-AD. The GAL4-DNA-BD fused to 
a protein ' $\mathrm{X}$ ' and GAL4-AD fused to a protein ' $\mathrm{Y}$ ' to form the bait and the target of the interaction trap, respectively. A selection of host cells with different reporter genes and different growth selection markers provide a means to detect and confirm protein-protein interactions and has significantly fewer false positives (13-16).

In this way, the bait plasmid pGBKT7-MHBs was transformed into the AH109 yeast strain. The MHBs gene was expressed in the yeast cells. Subsequent to the bait plasmid pGBKT7-MHBsAH109 yeast strain being mated with the liver cDNA library Y187 yeast strain, the diploid yeast cells were plated on QDO media containing X- $\alpha$-gal and two true positive colonies were obtained. By sequence analysis of isolated library plasmids, two gene sequences with known functions were obtained; one of them was termed the Human DNA sequence from clone RP11-490D19 on chromosome 9 (contains the ZNF189 gene for zinc finger protein 189). Notably, the present study screened Homo sapiens 12 BAC RP11-180M15 (Roswell Park Cancer Institute Human BAC Library) interacting with MHBs protein from the liver cDNA library.

These interacting proteins screened by yeast two-hybrid were closely correlated with the occurrence and development of different types of tumor. Identifying these interacting proteins may provide novel insights into the biological functions of the MHBs protein, the pathogenesis of HBV and the causes of malignancy conversion. Further experiments are required to elucidate how the interactions between MHBs protein and the aforementioned interacting proteins affect the occurrence and development of chronic hepatitis B, hepatic fibrosis and hepatocarcinoma.

\section{Acknowledgements}

The authors would like to thank the Institute of Infectious Diseases, Ditan Hospital (Beijing, China) for partial financial and technological assistance in conducting this study.

\section{References}

1. Murakami S: Hepatitis B virus X protein: structure, function and biology. Intervirology 42: 81-99, 1999.

2. Kekulé AS, Lauer U, Meyer M, Caselmann WH, Hofschneider PH and Koshy R: The preS2/S region of integrated hepatitis B virus DNA encodes a transcriptional transactivator. Nature 343: 457-461, 1990

3. Hildt E, Saher G, Bruss V and Hofschneider PH: The hepatitis B virus large surface protein (LHBs) is a transcriptional activator. Virology 225: 235-239, 1996.

4. Hildt E, Urban S and Hofschneider PH: Characterization of essential domains for the functionality of the MHBst transcriptional activator and identification of a minimal MHBst activator. Oncogene 11: 2055-2066, 1995.

5. Bruss V, Lu X, Thomssen R and Gerlich WH: Post-translational alterations in transmembrane topology of the hepatitis B virus large envelope protein. EMBO J 13: 2273-2279, 1994.

6. Matsumoto M, Hsieh TY, Zhu N, VanArsdale T, Hwang SB, Jeng KS, Gorbalenya AE, Lo SY, Ou JH, Ware CF and Lai MM: Hepatitis $C$ virus core protein interacts with the cytoplasmic tail of lymphotoxin-beta receptor. J Virol 71: 1301-1309, 1997.

7. Mahoney FJ: Update on diagnosis, management, and prevention of hepatitis B virus infection. Clin Microbiol Rev 12: 351-366, 1999.

8. Dong $\mathrm{Z}$ and Cheng J: Study on definition of pre-X region in hepatitis B virus genome. Shijie Huaren Xiaohua Zazhi 8: 1097-1101, 2003

9. Tong S, Kim KH, Chante C, Wands J and Li J: Hepatitis B virus e antigen variants. Int J Med Sci 2: 2-7, 2005.

10. Shao J, Li Y, Li H, Wu Q, Hou J and Liew C: Deletion of chromosomes $9 \mathrm{p}$ and 17 associated with abnormal expression of p53, p16/MTS1 and p15/MTS2 gene protein in hepatocellular carcinomas. Chin Med J (Engl) 113: 817-822, 2000.

11. Zhao S, Malek J, Mahairas G, Fu L, Nierman W, Venter JC and Adams MD: Human BAC ends quality assessment and sequence analyses. Genomics 63: 321-332, 2000.

12. Emmert-Buck MR, Gillespie JW, Pawletz CP, Ornstein DK, Basrur V, Appella E, Wang QH, Huang J, Hu N, Taylor P and Petricoin EE III: An approach to proteomic analysis of human tumors. Mol Carcinog 27: 158-165, 2000.

13. Fields S and Song O: A novel genetic system to detect protein-protein interactions. Nature 340: 245-246, 1989.

14. Osman A: Yeast two-hybrid assay for studying protein-protein interactions. Methods Mol Biol 270: 403-422, 2004.

15. Gietz RD and Woods RA: Screening for protein-protein interactions in the yeast two-hybrid system. Methods Mol Biol 185: 471-486, 2002.

16. Zhen Z: Progress in proteomics. Sheng Wu Gong Cheng Xue Bao 17: 491-493, 2001 (In Chinese). 